\title{
PROPER EFFICIENT POINTS FOR MAXIMIZATIONS WITH RESPECT TO CONES*
}

\author{
J. BORWEIN†
}

\begin{abstract}
Proper efficient points (Pareto maxima) are defined in tangent cone terms and are characterized by the existence of equivalent real-valued maximization problems.

1. Introduction. Suppose that $X$ and $Y$ are (locally) convex (topological vector) spaces over $R$ and that $S \subset Y$ is a nontrivial closed convex cone which induces a partial ordering $\leqq_{s}$. The vector maximization problem for $f$ mapping $X$ into $Y$ and $A \subset X$,
\end{abstract}

$$
\max f(x) \quad \text { subject to } x \in A \quad(\mathrm{VMP})
$$

is the problem of finding all efficient points $\bar{x} \in A: \bar{x}$ is said to be efficient (Pareto optimal) if $x \in A$ and

$$
f(x) \geqq_{s} f(\bar{x}), \quad f(x) \neq f(\bar{x}) \quad \text { implies that } \bar{x} \notin A .
$$

Geffrion [1] has studied this problem in finite dimensions with the coordinate ordering and has suggested a restriction to "proper" efficient points which allows for a reasonable characterization. Kuhn and Tucker [4] have also used the term but their notion requires differentiability and appears too broad for satisfactory analysis (see below).

This paper proposes a general notion of properness which is defined in terms of tangent cones as developed by Varaiya [8], Guignard [2], Zlobec [9] and others and which coincides with Geffrion's definition in the central case.

2. Preliminaries. Throughout the paper all spaces are assumed Hausdorff and convex and "ฏ," is the partial order induced by $S$.

Definition 1. Suppose $C \subset X$ and $\bar{x} \in \bar{C}$. The tangent cone to $C$ at $\bar{x}$ is defined to be the set of limits of the form $h=\lim t_{n}\left(x_{n}-\bar{x}\right)$ with $\left\{t_{n}\right\}$ a sequence of nonnegative real numbers and $\left\{x_{n}\right\} \subset C$ a sequence with limit $\bar{x}$. It is denoted $T(C, \bar{x})$.

When $X$ is metrizable, $T(C, \bar{x})$ is closed. It is always a nonempty cone containing 0 , but need not be closed in general spaces unless defined in terms of nets which leads to other embarrassments. The closed convex hull of $T(C, \bar{x})$ is called the pseudo-tangent cone and is denoted by $P(C, \bar{x})$. Various properties of pseudo-tangent cones can be found in [2], [8], [9] and a forthcoming paper of the author.

DEFINITION 2. A point $\bar{x}$ will be said to be a proper efficient point of (VMP) if it is efficient and

$$
\bar{T}(f(A)-S, f(\bar{x})) \cap S=0 .
$$

\footnotetext{
* Received by the editors March 5, 1975, and in final revised form April 14, 1975.

$\uparrow$ Department of Mathematics, Dalhousie University, Halifax, Nova Scotia, Canada. This research was supported in part by National Research Council Grants A7751 and A7675.
} 
Definition 3. When $f(x)=\left(f_{1}(x), \cdots, f_{p}(x)\right)$ maps $R^{k}$ into $R^{p}$, Geffrion defines $\bar{x}$ to be properly efficient with respect to the coordinate ordering if it is efficient and if there is some real $M>0$, such that for each $i$ one has

$$
\frac{f_{i}(x)-f_{i}(\bar{x})}{f_{j}(\bar{x})-f_{j}(x)} \leqq M
$$

holding for some $j$ with $f_{j}(x)<f_{j}(\bar{x})$, whenever $x \in A$ and $f_{i}(x)>f_{i}(\bar{x})$.

It is a simple matter to verify that in this later framework, $(1)$ is a weaker requirement on (VMP) than (2) and, in fact, that when $f$ is continuous (1) implies the local efficiency of $\bar{x}$ with respect to the coordinate ordering.

Proposition 1. Suppose $\bar{x}$ is Geffrion proper efficient for $f$ over A. Then $\bar{x}$ satisfies

$$
T\left(f(A)-R^{n+}, f(\bar{x})\right) \cap R^{n+}=0 .
$$

Proof. Suppose $k \neq 0 \in R^{n+} \cap T\left(f(A)-R^{n+}, f(\bar{x})\right)$. Without loss of generality one may assume that $k_{1}>1, k_{i} \geqq 0, i=2, \cdots, n$. Let

$$
t_{n}\left(f\left(x_{n}\right)-r_{n}^{+}-f(\bar{x})\right) \rightarrow k,
$$

where $r_{n}^{+} \in R_{n}^{+}, \mathrm{t}_{\mathrm{n}} \geqq 0$ and $f\left(x_{n}\right)-r_{n}^{+} \rightarrow f(\bar{x})$ with $x_{n} \in A$. By choosing a subsequence one can assume that

$$
\tilde{I}=\left\{i \mid f_{i}\left(x_{n}\right)<f_{i}(\bar{x})\right\}
$$

is constant for all $n$ (and nonempty since $\bar{x}$ is Pareto efficient). Set $M>0$. Then for $n \geqq n_{0}$,

$$
\begin{aligned}
& f_{1}\left(x_{n}\right)-f_{1}(\bar{x}) \geqq t_{n / 2}^{-1}, \\
& f_{i}\left(x_{n}\right)-f_{i}(\bar{x}) \geqq-t_{n}^{-1} / 2 M .
\end{aligned}
$$

Then for all $i \in \tilde{I}$, one has

$$
0<f_{i}(\bar{x})-f_{i}\left(x_{n}\right) \leqq t_{n}^{-1} / 2 M,
$$

and for $n \geqq n_{0}$,

$$
\frac{f_{1}\left(x_{n}\right)-f_{1}(\bar{x})}{f_{i}(\bar{x})-f_{i}\left(x_{n}\right)} \geqq \frac{t_{n / 2}^{-1}}{t_{n}^{-1} / 2 M}=M,
$$

which contradicts Geffrion's definition.

Definition 4. Suppose $X^{\prime}$ is the topological dual of $X$. The dual cone $K^{+}$of a convex cone $K \subset X$ is defined by

$$
K^{+}=\left\{x^{\prime} \in X^{\prime} \mid x^{\prime}(x) \geqq 0, \forall x \in K\right\},
$$

while the dual cone $\left(K^{\prime}\right)^{+}$of a convex cone $K^{\prime} \subset X^{\prime}$ is defined by

$$
\left(K^{\prime}\right)^{+}=\left\{x \in X \mid x^{\prime}(x) \geqq 0, \forall x \in K^{\prime}\right\} .
$$

It follows from these definitions that (i) $K^{+}$is weakly* closed; (ii) $\left(K^{+}\right)^{+}=\bar{K}$; (iii) $\left(\bar{K}_{1} \cap \bar{K}_{2}\right)^{+}=\operatorname{cl}\left(K_{1}^{+}+K_{2}^{+}\right)$(with closure in the weak* topology). $K^{+}$is welldefined even if $K$ is not a convex cone. In this case $\left(K^{+}\right)^{+}$is the closed convex hull of $K($ denoted $[\bar{K}])$. 
These facts all hold in convex spaces. Proofs can be found for normed spaces in [7].

3. Geometric motivation. The main aim of Definition 2 is to provide a notion of properness which can be applied when the cone $S$ is not the orthant ordering in $R^{n}$ and is not even polyhedral. Consider (VMP):

$$
\max _{x \in A} f(x)=\max _{z \in f(\boldsymbol{A})} z=\max _{z \in f(\boldsymbol{A})-S=E} z .
$$

This last equivalence is introduced so that, in the case that $f$ is concave with respect to $S$ and $A$ is convex, the optimization in the image space is still a concave problem. Definition 2 says that $\bar{x}$ is proper when, with $\bar{z}=f(\bar{x})$, one has

$$
T(E, \bar{z}) \cap S=0 .
$$

In general, then, the concept of properness is an attempt to remove those efficient points which can be approached in directions which point into $S$. In the case that $S=R^{n+}$, this can be done by considering the components separately; in more general orderings a more technical notion of direction must be introduced. This is done herein with tangent cones. Consider the following examples.

Example 1. Let $X=R^{3}, S=\left\{x \mid x=\left(x_{1}, x_{2}, x_{3}\right), x_{3} \geqq 0, x_{1}^{2}+x_{2}^{2} \leqq x_{3}^{2}\right\}$. Let $A=\{x \mid\|x\| \leqq 1\}$ and let $f=I$. The efficient points for (VMP) are $\{x \mid\|x\|=1, x \in$ $S\}$. Since $E=A-S$ is convex, $T(E, f(\bar{x}))$ is the smallest closed convex cone containing $E$ with vertex at $f(\bar{x})=\bar{x}$. It is easily seen that for those $x$ with $\|x\|=1$, $x \in S$ and $x_{1}^{2}+x_{2}^{2}=x_{3}^{2}$ (or $x_{3}=\frac{1}{\sqrt{2}}, x_{1}^{2}+x_{2}^{2}=\frac{1}{2}$ ), this cone has a boundary ray in common with $S$; while for any other efficient $x$ this cannot happen. In this case the efficient improper points form the relative boundary of the efficient points on $A$.

Example 2. Let $X=R^{2}, \quad S=\left\{x \mid x_{2} \geqq 0, x_{1} \geqq x_{2}\right\}$. Let $A=$ $\left\{\left(x_{1}, x_{2}\right) \mid x_{1}^{2}+x_{2}^{2} \leqq 1, x_{1} \geqq 0, x_{2} \geqq 0\right\}$ and let $f=I$. The efficient points are those $x$ on the arc in $A$ for which $x_{1}^{2}+x_{2}^{2}=1$. Again $T(f(A)-S, f(x))$ is the smallest closed convex cone containing $A-S$ at $f(x)$. This only intersects $S \mid\{0\}$ when $x=f(x)$ lies at the upper endpoint of the arc. There is, therefore, only one improper point $(0,1)$.

Example 3. Consider $X, S, f$ as in the previous example, and let $A_{1}=$ $A \cap\left\{\left(x_{1}, x_{2}\right) \mid x_{1} \leqq \frac{1}{2}\right.$ or $\left.x_{2}=0\right\}$. The efficient points are now

$$
\{(1,0)\} \bigcup\left\{\left(x_{1}, x_{2}\right) \mid x_{1} \leqq \frac{1}{2}, x_{1}^{2}+x_{2}^{2}=1\right\} \bigcup\left\{\left(\frac{1}{2}, x_{2}\right) \mid x_{2} \geqq 0, x_{2} \leqq \frac{1}{2}\right\}
$$

The problem is no longer convex, and $T(A-S, f(x))$ is easily calculated. Only $\left(\frac{1}{2}, 0\right)$ and $(0,1)$ are improper.

We see that properness gives us a criterion for excluding some efficient points (those which can be "approached from within $S$ ") for which, as will be shown, equivalent real maximizations fail to exist.

4. Some cone separation theorems. It is necessary to establish two abstract separation theorems for convex cones before proving general multiplier theorems for (VMP).

Proposition 2. Suppose N, S are closed convex cones in $X$ and that $N \cap S=0$. Suppose that the dual cone $S^{+}$has nonempty interior in some topology $\tau$ which gives 
$X$ as the dual of $X^{\prime}$. Then there is some $s^{+} \in\left(S^{+}\right)^{0}$ with $-s^{+} \in N^{+}$and

$$
s^{+}(s)>0 \quad \forall s \in S /\{0\} \text {. }
$$

In fact this last condition is equivalent to $s^{+} \in\left(S^{+}\right)^{0}$.

Proof. Using property (iii) under Definition 4 one sees that

$$
\{0\}^{+}=X^{\prime}=(N \cap S)^{+}=\tau-\operatorname{cl}\left(N^{+}+S^{+}\right),
$$

since $\tau$ is a topology of the dual pair $\left(X^{\prime}, X\right)$. Let $s^{\prime} \in\left(S^{+}\right)^{0}$. There is then some net $-s_{\alpha}^{\prime}=n_{\alpha}^{+}+s_{\alpha}^{+}$with $n_{\alpha}^{+} \in N^{+}, s_{\alpha}^{+} \in S^{+}$and $n_{\alpha}^{+}+s_{\alpha}^{+}$tending $(\tau)$ to $-s^{\prime}$. Since $-s^{\prime}$ is a $\tau$-interior point for $-S^{+}$, it follows that for $\alpha \geqq \alpha_{0}$,

$$
-s_{\alpha}^{\prime}=n_{\alpha}^{+}+s_{\alpha}^{+} \in-\left(S^{+}\right)^{0} \text {. }
$$

Thus $n_{\alpha}^{+}=-\left(s_{\alpha}^{\prime}+s_{\alpha}^{+}\right) \in-\left(S^{+}\right)^{0}-S^{+} \subset-\left(S^{+}\right)^{0}$. It follows that $n_{\alpha}^{+} \in N^{+}$and satisfies (3). Conversely, if $s^{+}$exists satisfying (3) and $\left(S^{+}\right)^{0} \neq \varnothing$, then

$$
S \cap\left\{x \mid s^{+}(x) \leqq 0\right\}=0
$$

and one can apply the previous argument to the two sets $S$ and $\left\{x \mid s^{+}(x) \leqq 0\right\}$ to derive that some $n^{+} \in N^{+}=\left\{x \mid s^{+}(x) \leqq 0\right\}^{+}$is also in $\left(-S^{+}\right)^{0}$. But $N^{+}=\bigcup_{\lambda \leqq 0} \lambda s^{+}$ and, since $0 \notin\left(S^{+}\right)^{0}, n^{+}=\lambda s^{+}, \lambda<0$, which implies that $s^{+} \in\left(S^{+}\right)^{0}$.

In particular, the theorem holds for any cone $S$ in $R^{n}$ which is pointed $(S \cap-S=0)$, since this means $S^{+} \subset R^{n}$ has nonempty interior. In the case that $\left(S^{+}\right)^{0}$ cannot be guaranteed nonempty, one can still prove the existence of $s^{+}$ satisfying (3) if one requires that $S$ have a compact base $B$.

Proposition 3. Suppose $N, S$ are closed convex cones in $X$ such that $N \cap S=$ 0. Suppose that $S \cap-S=0$ and that $S$ is locally compact (has a compact neighborhood base in the relative topology on $S$ ). Then one can find $s^{+} \in-N^{+}$ satisfying (3).

Proof. The local compactness condition on $S$ guarantees by [3, (2.4)] that one can find a compact convex subset $B$ of $S$, such that $0 \notin B$, with $S=\cup_{\lambda \geqq 0} \lambda B$.

It follows that $B$ and $N$ can be strictly separated [5] and that there is some $s^{+} \in X^{\prime}$ with

$$
s^{+} \in-N^{+} \text {and } s^{+}(x)>0 \quad \forall x \in B
$$

It follows immediately that $s^{+}(s)>0 \forall s \in S /\{0\}$.

Remark. It is easy to see that in a locally convex space a pointed cone $S$ is locally compact exactly when it has a compact generating base. That is: $S=$ $\cup_{\lambda \geqq 0} \lambda B$ where $B$ is compact, convex and $0 \notin B$.

5. Equivalences. One can now derive the basic characterization of proper efficient points.

THEOREM 1. Suppose that $\bar{x}$ is optimal for

$$
\max s^{+} f(x) \quad \text { subject to } x \in A \quad\left(P\left(s^{+}\right)\right)
$$

and that $s^{+}$satisfies (3). Then $\bar{x}$ is a proper efficient point.

Proof. Suppose $h \in T(f(A)-S, f(\bar{x}))$. Then

$$
h_{n}=t_{n}\left(f\left(x_{n}\right)-s_{n}-f(\bar{x})\right) \rightarrow h
$$


with $t_{n} \geqq 0, f\left(x_{n}\right)-s_{n} \rightarrow f(\bar{x}), x_{n} \in A, s_{n} \in S$. For each $n, s^{+} f\left(x_{n}\right) \leqq s^{+} f(\bar{x})$ since $\bar{x}$ is optimal for $\left(P\left(s^{+}\right)\right)$and so

$$
\lim t_{n}\left(s^{+}\left(f\left(x_{n}\right)-s_{n}\right)-s^{+} f(\bar{x})\right) \leqq 0 .
$$

It follows that $s^{+}(h) \leqq 0 \forall h \in \bar{T}(f(A)-S, f(\bar{x}))$. Were $h$ to belong to $S /\{0\}$, one would have $s^{+}(h)>0$ since (3) holds. This is impossible and $\bar{T}(f(a)-S, f(\bar{x})) \cap$ $S=0$.

It is clear that if $-x$ were not efficient and $x_{1} \in A$ with $f\left(x_{1}\right) \geqq_{s} f(\bar{x})$, that the definition of $s^{+}$would imply that $s^{+} f\left(x_{1}\right)>s^{+} f(\bar{x})$ which contradicts the optimality of $\bar{x}$ for $\left(P\left(x^{+}\right)\right)$.

THEOREM 2. Suppose that $f$ is concave with respect to $S$ and that $A$ is convex. Suppose $X$ and $S$ satisfy the hypotheses of Proposition 2 or 3. Then $\bar{x}$ is properly efficient for (VMP) if and only if $\bar{x}$ is optimal for $\left(P\left(s^{+}\right)\right)$for some $s^{+}$satisfying (3).

Proof. Sufficiency was proved in Theorem 1 . Suppose now that $\bar{x}$ is properly efficient. Since $f$ is concave and $A$ is convex, $f(A)-S=\{z \mid f(x) \geqq z, x \in A\}$ is convex. An elementary proposition in [3] shows that in this case,

$$
f(A)-S-f(\bar{x}) \subset \bar{T}(f(A)-S, f(\bar{x}))=N
$$

and that $N$ is convex. Because $\bar{x}$ is assumed proper, $N \cap S=0$. Since either Proposition 2 or 3 holds, $s^{+}$satisfying (3) exists with $-s^{+} \in N^{+}$. In particular, since (4) holds,

$$
s^{+}(f(x)-s-f(\bar{x})) \leqq 0 \quad \forall x \in A, \quad s \in S .
$$

Setting $s=0$ shows that $\bar{x}$ is optimal for $\left(P\left(s^{+}\right)\right)$with $s^{*}(s)>0 \forall s \in S /\{0\}$.

In finite dimensions with coordinate ordering, this equivalence is exactly the same as Geffrion's. Thus for coordinate concave programs, Definitions 2 and 3 coincide. It is worth noting that the use of the set $\bar{T}(f(A)-S, f(\bar{x}))$ rather than the smaller $\bar{T}(f(A), f(\bar{x}))$ is motivated by the need for (4) to hold. If one desires the equivalence of Theorem 2 only for problems with $f(A)$ convex (which includes $A$ convex, $f$ linear) one need only require that

$$
\bar{T}(f(A), f(\bar{x})) \cap S=0 .
$$

Example. $f_{1}(x)=\left(-x^{2}, x, x\right)$ is an example of a coordinate concave function satisfying (1) or (2) on $R^{n}$ at $0 ; f_{2}(x)=\left(-x^{2}, x, 0\right)$ does not. This can be seen either directly from Definition 2 or from the respective presence and absence of positive multipliers when one applies Theorem 2 .

If the hypotheses of Theorem 2 hold and the convex feasible set $A$ is, in fact, $\left\{x \mid g(x) \geqq_{B} 0, x \in C\right\}$ for some function $g$ mapping $X$ into $Z$, concave with respect to $B$, and some convex $C$, one has the following "Lagrange" multiplier theorem.

THeOREM 3. Suppose $B$ is a convex cone with interior and that $g\left(x_{0}\right) \in B^{0}$. Suppose $\bar{x}$ is a proper efficient point for (VMP) with $A=\{x \mid g(x) \geqq 0, x \in C\}$. Then there is some continuous linear mapping $T$ of $Z$ into $Y$ such that $T(B) \subset S$ and $\operatorname{Tg}(\bar{x})=0$ with $\bar{x}$ properly efficient for the unconstrained concave problem

$$
\max f(x)+\operatorname{Tg}(x) \quad \text { subject to } x \in C \quad \text { (UCP). }
$$

Proof. Apply Theorem 2 to produce $s^{+}$satisfying (3) with

$$
s^{+} f(\bar{x})=\max s^{+} f(x) \quad \text { subject to } g(x) \geqq 0, \quad x \in C .
$$


The standard Lagrange multiplier theorem ([9]) guarantees that $u^{+} \in B^{+}$ exists with $u^{+} g(\bar{x})=0$ and

$$
s^{+} f(\bar{x}) \geqq s^{+} f(x)+u^{+} g(x) \quad \forall x \in C .
$$

Choose $\mathrm{s} \in \mathrm{S}$ with $s^{+}(s)=1$. Let $T_{0}: Z \rightarrow Y$ be defined by $T_{0}(z)=u^{+}(z) s$. Then $T_{0}(B) \subset S, T_{0}$ is continuous, linear and $T_{0} g(\bar{x})=0$. Equation (6) can be rewritten as

$$
s^{+}\left(f(x)+T_{0} g(x)\right) \leqq s^{+}\left(f(\bar{x})+T_{0} g(\bar{x})\right), \quad x \in C,
$$

from which it follows, using Theorem 2 again, that $\bar{x}$ is a proper efficient point for (UCP) with $T=T_{0}$.

6. Differential conditions. Consider now the Pareto maximization problem

$$
\max f(x) \quad \text { subject to } g(x) \in B, \quad x \in C \quad(\mathrm{P}),
$$

where $f: X \rightarrow Y, g: X \rightarrow Y$ are Fréchet differentiable functions between normed spaces and $C \subset X, B \subset Z$ are arbitrary sets.

DEFINITION 5. The generalized constraint condition on $g$ is said to hold at $\bar{x}$ if there is some closed convex cone $G$ such that $G \cap K \subset T(A, \bar{x})$, where $K=$ $\left\{h \mid g^{\prime}(\bar{x})(h) \in P(B, g(\bar{x}))\right\}$.

(This is necessarily slightly stronger than Zlobec's condition [9] in which $P(A, \bar{x})$ replaces $T(A, \bar{x})$.) As before, $A$ denotes $g^{-1}(B) \cap C$.

Definition 6 [4]. Suppose $K$ and $G$ satisfy the constraint condition. $H(G)$ is said to hold when

(a) $K^{+}+G^{+}$is closed,

(b) $H=\left\{u^{+} \cdot g^{\prime}(\bar{x}) \mid u^{+} \in P^{+}(B, g(\bar{x}))\right\}$ is closed, (in the weak* topology).

$H(G)$ is satisfied in particular when $K, G, B$ are polyhedrally convex in finite dimensions. The author in his thesis has given fairly general conditions for $H(G)$ to hold.

THEOREM 4. Suppose $\bar{x}$ is a (local) proper efficient point for (P) and that $G$ satisfies the generalized constraint qualification with $H(G)$ holding. Suppose either $\left(S^{+}\right)^{0} \neq \varnothing$ or that $S$ is pointed and has a compact base. There is some $s^{+} \in S^{+}$with $s^{+}(s)>0$ if $s \in S /\{0\}$, and some $u^{+} \in P^{+}(B, g(\bar{x}))$ such that

$$
s^{+} f^{\prime}(\bar{x})-u^{+} g^{\prime}(\bar{x}) \in-G^{+} \text {. }
$$

Proof. By hypothesis, $S \cap \bar{T}(f(A)-S, f(\bar{x}))=0$. It is an elementary property of tangent cones that.

$$
f^{\prime}(\bar{x})(\bar{T}(A, \bar{x})) \subset \bar{T}(f(A), f(\bar{x})) .
$$

Combining these two containments with $K \cap G \subset \bar{T}(A, \bar{x})$, one sees that $\operatorname{cl}\left(f^{\prime}(\bar{x})(K \cap G)\right) \cap S=0$.

(This last containment is essentially Kuhn and Tucker's notion of properness if one takes $\left.S=R^{n+}\right)$. All the hypotheses of Proposition 2 or 3 are met with $N=\operatorname{cl}\left(f^{\prime}(\bar{x})(K \cap G)\right)$. There is some $s^{+}$satisfying (3) with $s^{+} f^{\prime}(\bar{x})(h) \leqq 0 \forall h \in$ $K \cap G$. This means

$$
s^{+} f^{\prime}(\bar{x}) \in-(K \cap G)^{+}=-\left(K^{+}+G^{+}\right)
$$


(using $H(G)$ and property (iii) of Definition 4). A straightforward separation argument shows that $\bar{H}=K^{+}$. This combined with $H(G)$ and (8) yields

$$
s^{+} f^{\prime}(\bar{x})+u^{+} g^{\prime}(\bar{x}) \in-G^{+},
$$

where $s^{+}(s)>0$ if $s \in S /\{0\}$ and $u^{+} \in P^{+}(B, g(\bar{x}))$.

In the standard finite-dimensional programming problem, $C, B$ are coordinate cones and the Kuhn-Tucker constraint condition implies that $K \cap P(C, \bar{x}) \subset$ $T(A, \bar{x})$. This means that Theorem 4 includes the Pareto maximization of any such program with respect to any pointed cone in $R^{n}$. Thus one sees that Geffrion's first order necessary condition is subsumed by Theorem 4 .

As in the case of real-valued objective functions, weak sufficiency conditions can be described for (P) using the theory developed by Guignard [2].

In another direction if one does not require $H(G)$ to hold, one still has

$$
s^{+} f^{\prime}(\bar{x}) \in \operatorname{cl}\left(\bar{H}+G^{+}\right),
$$

which is much like Zlobec's asymptotic results in [9].

7. Conclusion. The paper provides a tangent cone definition of proper efficiency which coincides with Geffrion's for concave programs and coordinate orderings and which enables one to develop the theory in a much more general framework. It seems possible that some requirement of properness could be fruitfully imposed on various other notions of maximization allowing one to characterize various classes of extreme points in tangent cone terms. Using compact derivatives [10] one can extend the results to arbitrary convex spaces.

Acknowledgments. This paper was partially prepared while the author was working on his D. Phil under Dr. M. A. H. Dempster of Balliol College, Oxford. Without his continued interest and direction it would not have been written.

\section{REFERENCES}

[1] A. M. Geffrion, Proper efficiency and the theory of vector maximization, J. Math. Anal. Appl., 22 (1968), pp. 618-630.

[2] M. GUIGNARD, Generalized Kuhn-Tucker conditions for mathematical programming problems in Banach space, this Journal, 7 (1969), pp. 232-241.

[3] V. L. KLEE, Separation properties of convex cones, Proc. Amer. Math. Soc., 6 (1955), pp. 313-318.

[4] H. W. KuHN AND A. W. TUCKER, Nonlinear programming, Proc. Second Berkeley Symp. on Mathematical Statistics and Probability, University of California Press, Berkeley, Calif., 1950, pp. 481-492.

[5] D. G. Luenberger, Optimization by Vector Space Methods, John Wiley, New York, 1969.

[6] A. Peressini, Ordered Topological Vector Spaces, Harper and Row, New York, 1967.

[7] K. RitTER, Optimization in linear spaces II, Math. Ann., 183 (1969), pp. 169-180.

[8] P. P. VARAIYA, Nonlinear programming in Banach spaces, SIAM J. Appl. Math., 19 (1967), pp. 239-244.

[9] S. ZLOBEC, Asymptotic Kuhn-Tucker conditions for mathematical programming in a Banach space, this Journal, 9 (1970), pp. 505-512.

[10] S. ZlOBEC AND H. MASSAM, Various definitions of the derivative in mathematical programming, presented at the VIII Internat. Symp. on Mathematical Programming, Stanford Univ., Palo Alto, Calif., 1973. 